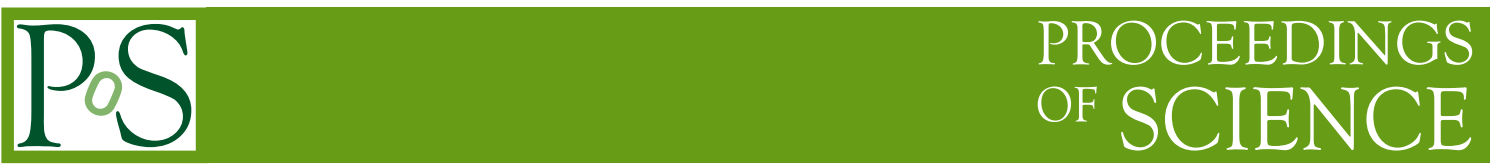

\title{
Beauty physics with heavy ions and prospects
}

\author{
Alessandro De Falco* \\ Università/INFN Cagliari, Italy \\ E-mail: alessandro.de.falcodca.infn.it
}

\begin{abstract}
Heavy flavours are generally considered one of the fundamental probes of the Quark-Gluon Plasma (QGP), the state of matter in which partons are deconfined, that can be created in relativistic heavy-ion collisions. Due to their large mass, heavy quarks are predominantly produced in hard scattering processes during the early stages of the collision lie Open heavy flavour measurements may probe the energy density of the system by means of the energy loss through elastic scatterings and gluon radiation. The radiative energy loss is predicted by QCD to be larger for gluons than for quarks, and light quark energy loss should be larger than that for heavy quarks due to the dead cone effect. The measurement of the yield of beauty hadrons can therefore shed light on the energy loss mechanism and on the mass hierarchy.

Quarkonia are another crucial probe of the QGP. They can melt in the medium due to color screening at a temperature which increases with the binding energy of the system, thus acting as a probe of the QGP temperature. Bottomonia are of particular interest in this respect, since they can provide a clear suppression pattern.

Beauty and bottomonia measurements in heavy-ion collisions at the LHC are reviewed. Results based on semi-leptonic decays of charm and beauty hadrons, non-prompt $\mathrm{J} / \psi$ from $\mathrm{B}$ decays and b-tagged jets in $\mathrm{Pb}$ - $\mathrm{Pb}$ collisions are discussed, as well as the measurements of $\Upsilon$ suppression at mid and forward rapidity provided by the CMS and ALICE experiments. Cold nuclear matter effects are investigated through the measurements in $\mathrm{p}-\mathrm{Pb}$ collisions.
\end{abstract}

The 15th International Conference on B-Physics at Frontier Machines at the University of Edinburgh, 14 -18 July, 2014

University of Edinburgh, UK

${ }^{*}$ Speaker. 


\section{Introduction}

High energy heavy-ion collisions provide a tool to study the quark-gluon plasma (QGP), a phase of strongly interacting matter in which partons are deconfined, that can be reached at high temperature and energy density. In this context, heavy quarks play an important role in the understanding of the properties of the deconfined medium since, due to their high mass, they are predominantly produced in the early phases of the collision with typical times of $0.05-0.15 \mathrm{fm} / c$, shorter than the QGP formation time $(\sim 1 \mathrm{fm} / c)$. They thus traverse the medium during its evolution and interact with its constituents losing energy through elastic scatterings and/or gluon radiation. The energy loss in the medium is expected to depend on color charge, being larger for gluons than for quarks. A suppression of gluon radiation by heavy quarks at low angles (the so-called dead cone effect) is predicted [1]. The effect is expected to be stronger for heavier quarks, thus leading to a mass hierarchy in the energy loss with $\Delta E_{b}<\Delta E_{c}<\Delta E_{\text {light quarks. The mechanism of energy }}$ loss is however not yet fully understood. Its dependence on the path length and medium density are still to be clarified.

At LHC energies, heavy flavour production is characterized by a cross section which is expected to be much higher than at RHIC, by a factor of about 5-10 for charm and up to 50 for beauty in pp collisions. The LHC is thus the ideal place to study heavy flavour production, allowing for accurate measurements in $\mathrm{Pb}-\mathrm{Pb}$ collisions, together with the reference provided by pp collisions. In order to disentangle cold nuclear matter (CNM) effects from the ones due to the hot and dense medium, data acquired in $\mathrm{p}-\mathrm{Pb}$ collisions are necessary, since in this system no QGP is expected to be formed.

Another fundamental probe of the deconfined medium is provided by quarkonia, both of the charmonium $\left(J / \psi, \psi^{\prime}\right)$ and bottomonium $\left(\Upsilon, \Upsilon^{\prime}, \Upsilon^{\prime \prime}\right)$ families. Quarkonium suppression due to color screening was predicted to be a signature for the formation of the QGP [2]. The suppression is expected to occur at temperatures higher than the critical temperature for the formation of the deconfined medium, the more tightly bound states being suppressed at higher temperatures [3]. A sequential suppression is thus expected for the quarkonium states. Bottomonia at the LHC provide a clearer signature of suppression since they are less affected than charmonia by possible effects of heavy quark recombination [4]. Moreover, they are expected to be less sensitive to cold nuclear matter effects [5].

In these proceedings, results on beauty and bottomonia production at the LHC are reviewed. Heavy flavour production measured through semi-leptonic decays of charm/beauty hadrons and the separation of the beauty contribution in the single electron channel will be shown. The $J / \psi$ from B decays will be compared to the results on charm production. Results on $\Upsilon$ suppression as a function of centrality will be shown at forward and mid-rapidity.

\section{Heavy flavour measurements}

Heavy flavour production can be studied through the semi-leptonic decays of charm and beauty hadrons. Measurements have been performed by the ALICE Collaboration in the muon channel at forward rapidity $[6,7]$ and in the electron channel at mid-rapidity $[8,9]$. In these measure- 

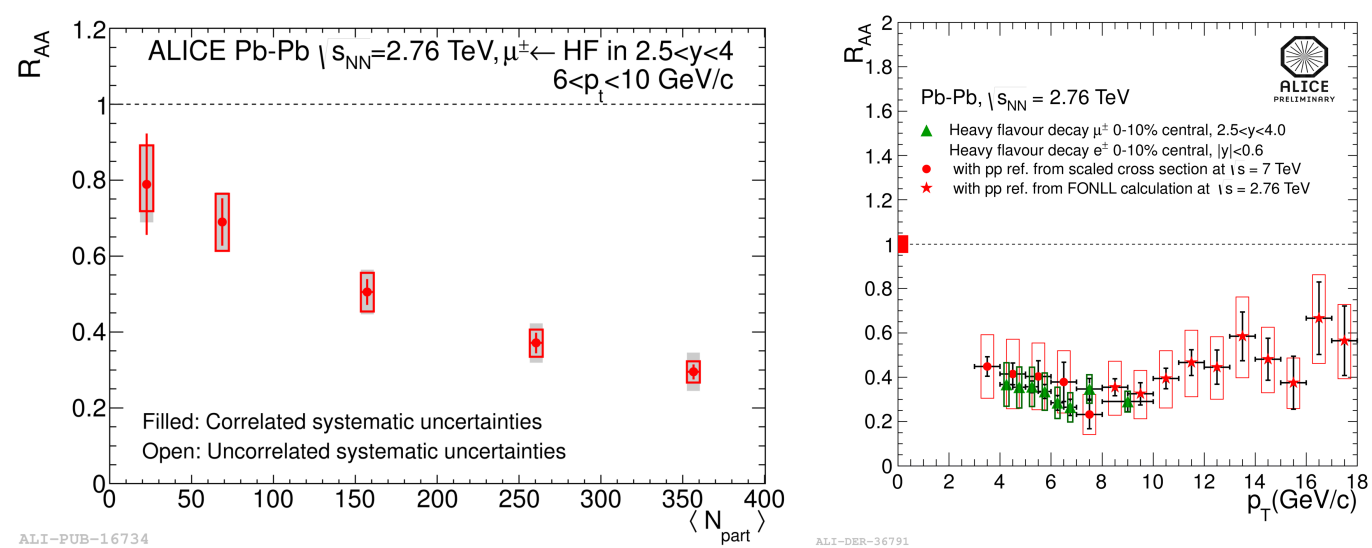

Figure 1: Left: $R_{A A}$ of muons from heavy flavour hadron decays in $\mathrm{Pb}-\mathrm{Pb}$ collisions as a function of $\left\langle N_{\text {part }}\right\rangle$, in $2.5<y<4$ and $6<p_{\mathrm{T}}<10 \mathrm{GeV} / c$. Right: $R_{A A}$ of muons and electron decays, at forward and mid-rapidity respectively, in central $\mathrm{Pb}-\mathrm{Pb}$ collisions, as a function of the transverse momentum.

ments, charm and beauty components are not separated, although it is possible to isolate the beauty contribution in the electron channel, as explained below.

The comparison between the results in $\mathrm{Pb}-\mathrm{Pb}$ and in pp collisions is obtained calculating the nuclear modification factor, defined as $R_{A A}\left(p_{\mathrm{T}}\right)=\frac{1}{\left\langle T_{A A}\right\rangle} \times \frac{d N_{A A} / d p_{\mathrm{T}}}{d \sigma_{p p} / d p_{\mathrm{T}}}$, where $\left\langle T_{A A}\right\rangle$ is the average nuclear overlap function in a given centrality class, calculated with the Glauber model [10], $d N_{A A} / d p_{\mathrm{T}}$ is the $p_{\mathrm{T}}$-differential yield in nucleus-nucleus collisions and $d \sigma_{p p} / d p_{\mathrm{T}}$ is the $p_{\mathrm{T}}$-differential cross section in pp collisions. The value of $R_{A A}$ is unity if a nucleus-nucleus collision can be treated as a superposition of independent nucleon-nucleon collisions. An $R_{A A}$ value lower than unity can arise from partonic energy loss as well as other nuclear effects.

The nuclear modification factor of muons from heavy-flavour hadron decays in $\mathrm{Pb}-\mathrm{Pb}$ collisions as a function of the mean number of participant nucleons $\left\langle N_{\text {part }}\right\rangle$ is shown in Fig. 1 (left) for $6<p_{\mathrm{T}}<10 \mathrm{GeV} / c$. A strong suppression of the $R_{A A}$ of muons from-heavy flavour hadron decays is observed, increasing with centrality and reaching a factor of about 3-4 in the $10 \%$ most central collisions. Similar results are obtained at midrapidity in the electron channel [8]. For central collisions, the $R_{A A}$ as a function of $p_{\mathrm{T}}$ at mid-rapidity for electrons and at forward rapidity for muons exhibit a similar magnitude and no significant dependence on $p_{\mathrm{T}}$ as shown in Fig. 1 (right).

Measurements in $\mathrm{p}-\mathrm{Pb}$ collisions provide an insight into the possible contribution of cold nuclear matter effects. In Fig. 2, the $R_{\mathrm{pPb}}$ for heavy flavour hadron decays into muons at forward (p-going direction) and backward (Pb-going direction) rapidity (left) and electrons at mid-rapidity (right) is shown as a function of $p_{\mathrm{T}}$ [11]. For comparison, the result in $\mathrm{Pb}-\mathrm{Pb}$ collisions is reported in the left plot. The results provide evidence that cold nuclear matter effects are small in the measured $p_{\mathrm{T}}$ range, indicating that the strong suppression observed in central $\mathrm{Pb}-\mathrm{Pb}$ collisions is a final state effect due to in-medium parton energy loss.

The measurement in the electron channel makes it possible to isolate the beauty contribution: due to their long lifetime ( $c \tau \sim 500 \mu \mathrm{m}$ ) beauty hadrons decay at a secondary vertex displaced with respect to the primary collision vertex, resulting in a broad distribution of the distance of closest approach (DCA) between the electron and the primary vertex, as shown in Fig. 3 (left). The nuclear modification factor in central $\mathrm{Pb}-\mathrm{Pb}$ collisions shows a suppression for $p_{\mathrm{T}}>3 \mathrm{GeV} / c$ (Fig. 3 

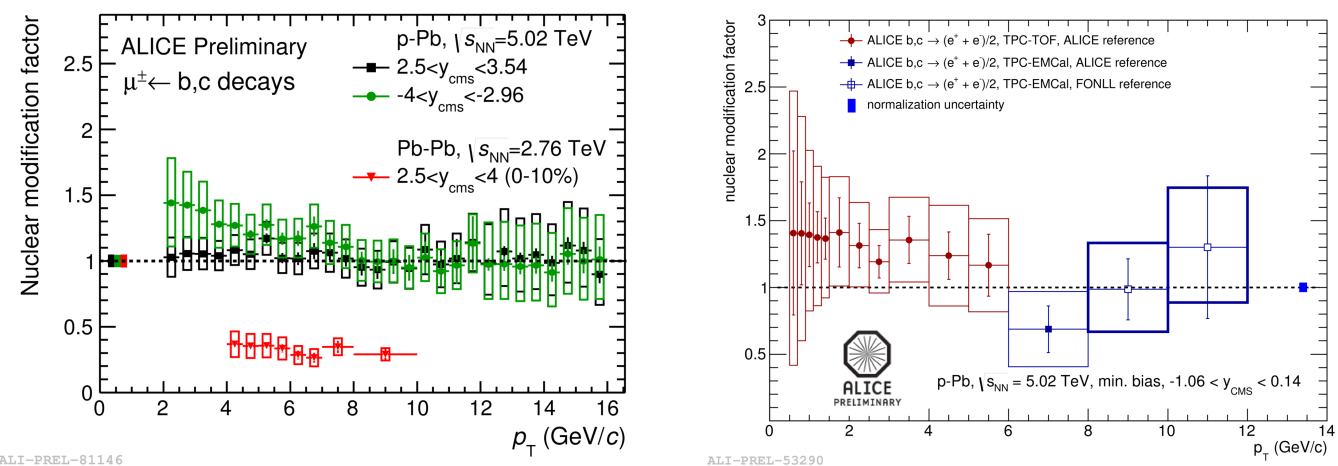

Figure 2: Left: Comparison of $p_{\mathrm{T}}$-differential nuclear modification factor of muons from heavy-flavour decays at forward $\left(2.5<y_{C M S}<3.54\right)$ and backward rapidity $\left(-4<y_{C M S}<-2.96\right)$ in $\mathrm{p}$-Pb collisions with the one measured in central $\mathrm{Pb}-\mathrm{Pb}$ collisions. Right: nuclear modification factor of electrons from heavy flavour hadron decays at mid-rapidity in $\mathrm{p}-\mathrm{Pb}$ collisions.
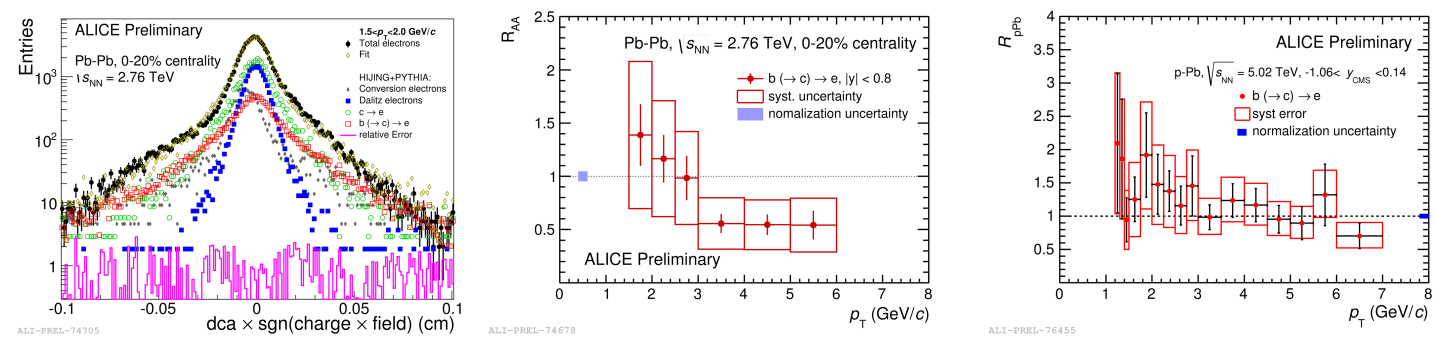

Figure 3: Inclusive electron distance of closest approach distribution (black points) fitted with templates for the various electron sources obtained from simulations (left). Nuclear modification factor of electrons from beauty hadron decays in central $\mathrm{Pb}-\mathrm{Pb}$ collisions (center) and in $\mathrm{p}-\mathrm{Pb}$ collisions (right).

center), although with large uncertainties. The same measurement performed in $\mathrm{p}-\mathrm{Pb}$ collisions (Fig. 3 right) gives an $R_{\mathrm{pPb}}$ value compatible with unity, although with large uncertainties. This result suggests that the effect observed in $\mathrm{Pb}-\mathrm{Pb}$ may be caused by the interaction with the hot medium, although the accuracy of the current results does not allow to draw firm conclusions.

A more direct measurement of beauty in $\mathrm{Pb}-\mathrm{Pb}$ collisions is performed through the detection of non-prompt $J / \psi$ mesons decaying into muon pairs. At LHC energies, the inclusive $J / \psi$ yield contains a sizeable contribution from B decays, which is characterized by a displacement of the $J / \psi$ decay vertex from the primary vertex, due to the relatively long lifetime of the B meson. Moreover, non-prompt $J / \psi$ s, being created after the QGP hadronization, should not suffer from color screening. The in-medium effects in this channel are thus related to the b-quark energy loss. Results obtained by the CMS [13] Collaboration on non-prompt $J / \psi$ are compared to the D meson measurements by ALICE [14] in Fig. 4. The $p_{\mathrm{T}}$ ranges are chosen such that the average $p_{\mathrm{T}}$ of the parent $\mathrm{B}$ mesons is compatible with the one of D mesons. For both D and B mesons the nuclear modification factor decreases from peripheral to central collisions with a similar suppression pattern, but with a $R_{A A}$ value for $\mathrm{B}$ mesons which is about two times larger than for $\mathrm{D}$ mesons. This result is consistent with the prediction of a mass hierarchy in the quark energy loss in the medium, which is expected to be lower for heavier quarks. 


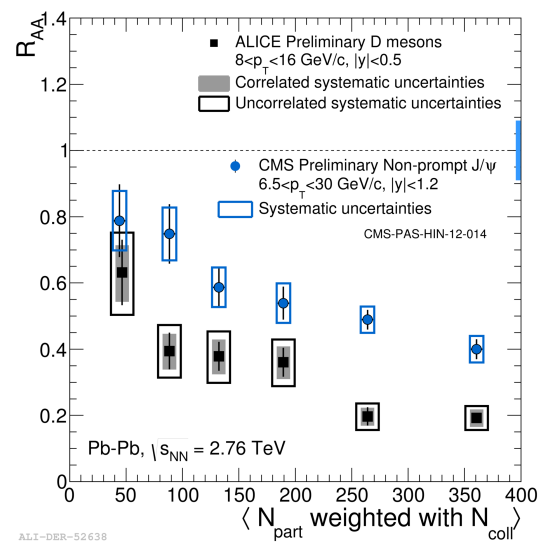

Figure 4: $R_{A A}$ for D mesons vs $N_{\text {part }}$ for $8<$ $p_{\mathrm{T}}<16 \mathrm{GeV} / c$ compared with non-prompt $\mathrm{J} / \psi$ $R_{A A}$ for $6.5<p_{\mathrm{T}}<30 \mathrm{GeV} / c$.

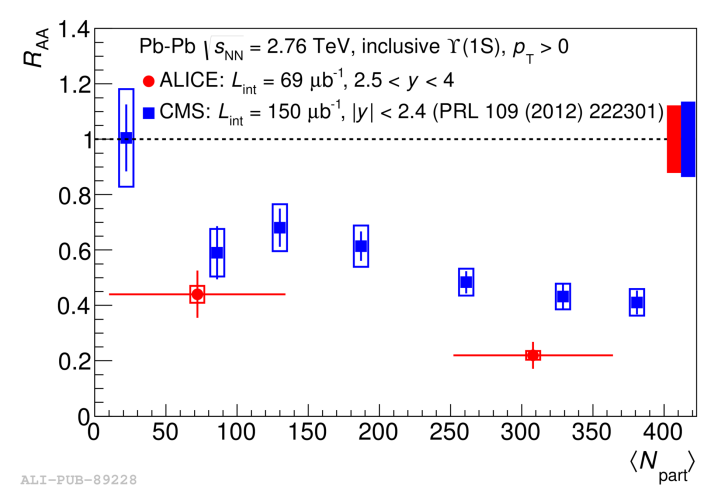

Figure 5: $\Upsilon(1 S) R_{A A}$ as a function of the average number of $\left\langle N_{\text {part }}\right\rangle$ as measured by CMS at midrapidity [18] and ALICE [20] at forward rapidity.

\section{3. $\Upsilon$ suppression}

The $\Upsilon$ inclusive cross section was measured in pp collisions in the dimuon decay channel by CMS [15] at mid-rapidity and by LHCb [16] and ALICE [17] at forward rapidity, with a wide rapidity coverage from the combined results of the three experiments $(|y|<4.5)$. The ALICE and $\mathrm{LHCb}$ results are in good agreement in the common rapidity region. The sequential suppression of the bottomonia states observed by the CMS experiment [18] is covered in [19], while in this proceedings the comparison between the results on $\Upsilon$ obtained by ALICE at forward rapidity [20] and the CMS results at mid-rapidity is discussed.

Results at mid-rapidity show a clear $\Upsilon$ suppression with respect to pp collisions that increases with the collision centrality (Fig. 5). The suppression is stronger at forward rapidity than at midrapidity. A comparison with theoretical models (not reported in these proceedings) shows that both the transport [21] and dynamical [22] models, that predict a suppression largely due to in-medium dissociation of higher mass bottomonia, underestimate the observed suppression at forward rapidity [20]. A precise measurement of $\Upsilon$ feed-down from higher mass bottomonia is needed in order to have more stringent comparisons with models. Also CNM effects have to be fully understood.

\section{Conclusions}

From the measurements of open heavy flavour production in $\mathrm{Pb}-\mathrm{Pb}$ and $\mathrm{pp}$ collisions performed so far, a clear indication for a substantial modification of the transverse momentum distribution emerged, resulting in a suppression of the yield of heavy-flavour hadrons at intermediate and high $p_{\mathrm{T}}$ in $\mathrm{Pb}-\mathrm{Pb}$ collisions relative to a binary-scaled pp reference. The suppression is stronger in central collisions than in peripheral ones, and does not show a significant rapidity dependence. p$\mathrm{Pb}$ collisions appear as a superposition of binary pp collisions, apart from possible small shadowing effects. This indicates that suppression in $\mathrm{Pb}-\mathrm{Pb}$ is a final state effect. Suppression for $\mathrm{D}$ mesons is stronger than for B mesons, in agreement with the expectation based on the dead cone effect. Combined information on D and B mesons will constrain predictions on energy loss and help to 
understand the energy loss mechanism and transport coefficients of the medium. Data collected on quarkonia show a clear $\Upsilon$ suppression that is stronger at forward rapidity than at mid-rapidity. Transport and dynamical models do not reproduce the trend as a function of $y$ and underestimate the suppression at forward rapidity. Improved beauty measurements in heavy-ion collisions will be made possible in LHC run 2 thanks to the increase in energy and integrated luminosity (by a factor 5-10 with respect to run 1), while in run 3 an upgrade of the ALICE Inner Tracking System and the addition of a Muon Forward Tracker will lead respectively to fully reconstructed beauty decays at mid-rapidity and to a separation of the non-prompt $J / \psi$ contribution at forward rapidity. Similar upgrades in ATLAS and CMS will allow improved b-tagging and full reconstruction of B meson decays.

\section{References}

[1] Yu.L. Dokshitzer and D.E. Kharzeev, Phys. Lett. B 519 (2001) 199.

[2] T. Matsui and H. Satz, Phys. Lett. B 178 (1986) 416.

[3] S. Digal, P. Petreczky and H. Satz, Phys. Rev. D 64 (2001) 094015.

[4] A. Andronic, P. Braun-Munzinger, K. Redlich and J. Stachel, Nucl. Phys. A 789 (2007) 334.

[5] R. Vogt, Phys. Rev. C 81 (2010) 044903.

[6] B. Abelev et al. (ALICE Collaboration), Phys. Lett. B 708 (2012) 265.

[7] B. Abelev et al. (ALICE Collaboration), Phys. Rev. Lett. 109 (2012) 112301.

[8] B. Abelev et al. (ALICE Collaboration), Phys. Rev. D 86 (2012) 112007.

[9] A. Festanti for the ALICE Collaboration, arXiv:1407.6541. M. Cacciari et al., JHEP 0407 (2004) 033.

[10] M. L. Miller, K. Reygers, S. Sanders, and P. Steinberg, Annu. Rev. Nucl. Part. Sci. 57 (2007) 205.

[11] L. Shuang for the ALICE Collaboration, arXiv:1408.1915.

[12] B. Abelev et al. (ALICE Collaboration), Phys. Lett. B 721 (2013) 13.

[13] CMS Collaboration, CMS PAS HIN-12-014

[14] D. Stocco for the ALICE Collaboration, J.Phys.Conf.Ser. 509 (2014) 012011.

[15] V. Khachatryan et al. (CMS Collaboration), Phys. Rev. D 83 (2011) 112004; V. Khachatryan et al. (CMS Collaboration), Phys. Lett. B 727 (2013) 101.

[16] R. Aaij et al. (LHCb Collaboration), Eur. Phys. J. C 72 (2012) 2025.

[17] B. Abelev et al. (ALICE Collaboration), Eur. Phys. J. C 74 (2014) 2974.

[18] S. Chatrchyan et al. (CMS Collaboration), Phys.Rev.Lett. 109 (2012) 222301.

[19] M.A. Nguyen, Heavy flavour production at CMS in heavy ion collisions, these proceedings.

[20] B. Abelev et al. (ALICE Collaboration), arXiv:1405.4493.

[21] L. Grandchamp, S. Lumpkins, D. Sun, H. van Hees, R. Rapp, Phys.Rev. C73 (2006) 064906; A. Emerick, X. Zhao, R. Rapp, Eur.Phys.J. A48 (2012) 72.

[22] M. Strickland, AIP Conf.Proc. 1520 (2013) 179. 